Deutsche Gesellschaft für Hämatologie und Onkologie e. V.

Geschäftsführender Vorsitzender Prof. Dr. Gerhard Ehninger

V.i.S.d.P. Mirjam Renz

Alexanderplatz 1, 10178 Berlin, Germany

Tel. +49 302787 6089-0, Fax -18

renz@dgho.de

www.dgho.de

\title{
Fachgesellschaft der Hämatologen und Onkologen fordert wirksamere Kontrollen und mehr Transparenz bei der Zubereitung von Zytostatika
}

In den letzten Tagen ging erneut ein Betrugsskandal mit Zytostatika für Chemotherapien durch die Presse. Onkologen, die Spezialisten für die Chemotherapie, beziehen jetzt Stellung und fordern eine bessere und transparentere Dokumentation. «Die Betrugsfälle der letzten Monate und die Prozesswelle zeigen, dass die vorhandenen Kontrollmechanismen nicht ausreichend sind», sagte Prof. Mathias Freund, Vorstandsmitglied der Deutschen Gesellschaft für Hämatologie und Onkologie e.V. (DGHO) im April in Berlin. «Der Gesetzgeber muss dringend sicher stellen, dass sowohl die siebenstellige Pharmazentralnummer als auch die Chargennummer der Zytostatika in den Begleitpapieren bei der Auslieferung der Zytostatika-Zubereitungen durch die Apotheker dokumentiert werden müssen», führt Prof. Gerhard Ehninger, geschäftsführender Vorsitzender der Fachgesellschaft aus. «Nur so können Betrügereien bei der Zubereitung durch den Apotheker sofort vom Arzt erkannt werden.»

Kliniken und Praxen sollen darüber hinaus verpflichtet werden, diese Unterlagen in der Krankenakte für 30 Jahre zu archivieren. «Chemotherapien sind auch heute noch mit einem gewissen Risiko behaftet», erläutert Freund. «Insbesondere müssen wir dabei an heute möglicherweise noch nicht bekannte Langzeitrisiken denken. Als Spezialisten für die Systemtherapie müssen wir deshalb sicherstellen, dass wir jederzeit nachvollziehen können, wo die Arzneimittel herkommen, die wir unseren Patienten geben.

\section{KARGER}

Fax +497614520714

Information@Karger.de

www.karger.com 